\title{
COVID-19 and Physical Activity Behaviour in People with Neurological Diseases: A Systematic Review
}

\section{Zuhal Abasıyanık $^{1,2} \mathbb{D} \cdot$ Merve Kurt $^{1,2} \mathbb{D} \cdot$ Turhan Kahraman $^{2} \mathbb{D}$}

Accepted: 21 January 2022 / Published online: 1 February 2022

(c) The Author(s), under exclusive licence to Springer Science+Business Media, LLC, part of Springer Nature 2022

\begin{abstract}
The COVID-19 pandemic has led to a radical lifestyle change, which may unintendedly change physical activity levels. We aimed to perform a systematic review to investigate the physical activity changes in people with neurological diseases, and to examine the relationship between physical activity and disease symptoms, and psychosocial factors. The review was performed in accordance with the Preferred Reporting Items for Systematic Reviews and Meta-Analyses (PRISMA) statement. A systematic search of the literature across five databases (PubMed, CINAHL, Web of Science, SCOPUS, and Cochrane Library) was carried out using the keywords relating to COVID-19, physical activity, sedentary behaviour, exercise, and the name of the neurological diseases. The systematic search was updated on 4 February 2021 with the same keywords. Fourteen studies $(n=7662$ persons with neurological diseases, $n=1663$ healthy controls) were eligible for this review. The study populations were Parkinson disease $(n=7)$, dementia $(n=1)$, multiple sclerosis $(n=1)$, spinal cord injury $(n=1)$, hereditary spastic paraplegia $(n=1)$, neuromuscular diseases $(n=1)$, Charcot-Marie-Tooth neuropathy $(n=1)$, and epilepsy $(n=1)$. Thirteen studies reported a decreased physical activity level, one study reported a high interruption rate of physiotherapy/rehabilitation. Furthermore, the physical activity reduction was associated with worse disease symptoms, depression, perceived health, and mental and physical components of quality of life. The COVID-19 pandemic has a negative impact on the physical activity levels of people with neurological diseases, and this change was related to the worsening of disease symptoms and psychosocial factors. Registration number A protocol of the review was registered with the PROSPERO database (CRD42020207676).
\end{abstract}

Keywords COVID-19 - Coronavirus · Physical activity · Sedentary behaviour . Exercise $\cdot$ Neurological Disease

Zuhal Abasıyanık

zuhalabasiyanik@gmail.com

Extended author information available on the last page of the article 


\section{Introduction}

Coronavirus Disease (COVID-19) has been declared as a global pandemic on 11 March 2020 by the World Health Organization (Jiménez-Pavón et al., 2020; Tison et al., 2020). To prevent or slow down the spread of COVID-19, the countries and territories have taken measures such as quarantine, closing schools and businesses, and banning cultural and sporting events to ensure social distance (Hammami et al., 2020; Jiménez-Pavón et al., 2020) These measures have led to a rapid change in lifestyle, and the physical activity level assessed by daily step counts have decreased compared to before the pandemic in the general population, which is an essential factor for physical and mental health (Tison et al., 2020; Tremblay et al., 2011) Neurological diseases are generally chronic and require using medications and rehabilitation services for a long time. Although there is no evidence that individuals with neurological diseases are more vulnerable to COVID-19, they may be more affected by healthcare disruptions, home confinement, and psychological stressors (FeriniStrambi \& Salsone, 2020) These factors can lead to more limitations of physical activity, either as routine physiotherapy or sports activities (Schirinzi et al., 2020; Woods et al., 2020).

Physical activity is any bodily movement produced by skeletal muscles that result in energy expenditure, and it improves the general health in both healthy population and people with neurological disabilities (Amekran \& el Hangouche, 2021; Boysen \& Krarup, 2009; Stennett et al., 2020) However, it has been well established that neurological diseases may negatively affect reaching a sufficient level of physical activity due to motor, sensory, and autonomic system involvement. Ultimately, a lack of physical activity causes deconditioning and worsening the disability (Ellis \& Motl, 2013; Elsworth et al., 2009; Stroud \& Minahan, 2009) However, it is known that physical activity improves the symptoms of neurological diseases and the quality of life of patients, so regular physical activity has been recommended as a first-line approach for many neurological diseases (Boysen \& Krarup, 2009; Goodwin et al., 2008; Stennett et al., 2020).

There are more varied physical activity opportunities outdoor (Jiménez-Pavón et al., 2020) However, in the period of the COVID-19 pandemic, the outdoor activities have been limited to prevent or slow down the spread of infection. The time spent at home has increased, and normal routines have been interrupted (Hammami et al., 2020; Jiménez-Pavón et al., 2020) Even so, there are many possibilities for physical activity that can be performed at home, but indoor activities may need more autonomy and motivation during a stressful time like a pandemic (Hammami et al., 2020) Following the emergence of the COVID-19 pandemic, there are many efforts to encourage people to be physically active. The American College of Sports Medicine published strategies to stay active at home (ACSM, 2020) Research papers have been released to provide activity suggestions, videobased physical activity suggestions have been made, and the importance of the issue was emphasized in the newspapers, and telehealth approaches have been 
used (Hammami et al., 2020; Jiménez-Pavón et al., 2020; Quinn et al., 2020; Reynolds, 2020).

The evidence has indicated that physical activity is a substantial factor for the health of persons with neurologic diseases and the efforts targeting to improve physical activity during the pandemic are important. Therefore, the objectives of this systematic review were to examine the impact of quarantine and isolation during the COVID-19 pandemic on the physical activity level and sedentary behaviour in people with neurological diseases, and the differences of physical activity levels and sedentary behaviours between people with neurological diseases and healthy controls. Secondly, we aimed to investigate the relationship between physical activity level and psychosocial factors, and disease symptoms.

\section{Methods}

A protocol of the review was registered with the PROSPERO database (CRD42020207676). The review was performed in accordance with the Preferred Reporting Items for Systematic Reviews and Meta-Analyses (PRISMA 2020) statement (Moher et al., 2009).

\section{Data Sources and Searches}

A systematic search for relevant articles was conducted on 22 September 2020 using electronic databases, namely, PubMed, CINAHL (EBSCO host), Web of Science (WOS), SCOPUS, and the Cochrane Library, independently by the two authors (ZA, MK) until 22 September 2020. The search terms for PubMed were: (((()((COVID19) OR (Novel Coronavirus)) OR (2019-nCoV)) OR (SARS-CoV-2)) OR (2019 novel coronavirus $))$ OR (SARS nCoV2)) AND ((()(((((stroke) OR (multiple sclerosis)) OR (Parkinson)) OR (brain injury)) OR (spinal cord injury)) OR (Alzheimer's disease)) OR (nervous system disease)) OR (neurological disorders)) OR (neurological diseases $)))$ AND ((((((physical activity) OR (exercise)) OR (sedentary)) OR (sedentary behaviour)) OR (leisure activity)) OR (sitting)) and this strategy was adapted in each database. Since the review topic is very current, the systematic search was updated on 4 February 2021 using the same keywords.

\section{Study Selection}

We used the Population, Intervention, Comparison, Outcome (PICO) framework as the criterion for inclusion of articles in this review (Santos et al., 2007) Population: adults with neurological disease including stroke, Parkinson's disease (PD), multiple sclerosis (MS), traumatic brain injury (TBI), spinal cord injury (SCI), Alzheimer's disease, and others. Intervention: quarantine and isolation during the COVID19 pandemic. Comparison: pre-pandemic physical activity level and sedentary 
behaviour or healthy controls. Outcomes: change in physical activity level or sedentary behaviour score from pre-pandemic to the last available follow-up after the pandemic, measured using the validated self-reported questionnaires or accelerometers/ pedometers.

The observational studies, including cross-sectional, cohort, and case-control studies published in English, were included. Initially, two authors independently screened articles based on title and abstract, but if the abstract did not provide sufficient information, the full text was read. Two authors (ZA, MK) independently reviewed the full text of papers for eligibility. In case of any indecision or disagreement, a third author (TK) was consulted.

We had excluded studies that involved pediatric neurological disorders (e.g., cerebral palsy and epilepsy). The animal studies, conference papers, study protocols, opinion papers, commentaries, case reports, systematic reviews, and meta-analysis were also excluded.

\section{Data Extraction and Quality Assessment}

National Heart, Lung, and Blood Institute (NHLBI) study quality assessment tool for observational cohort and cross-sectional studies was used to assess the methodological quality of the included articles. The tool has 14 items and is scored as yes, no, or cannot determine/not applicable/not reported (NHLBI, 2014).

Data extraction and data synthesis were conducted according to a guideline for systematic reviews (van Tulder et al., 2003) Two independent reviewers (ZA and MK) manually extracted key data from the included articles in a Summary of Findings table. The two reviewers applied pilot testing for the data extraction form together to minimize misinterpretation. The following data were placed in the appropriate place in table: date and place of study, study design including methodological quality, participants characteristics (a number of participants, age, sex, neurological condition), inclusion and exclusion criteria, measurement of physical activity, and sedentary behaviour, change of physical activity, and sedentary behaviour between pre-pandemic and during the pandemic or differences between persons with a neurological condition and healthy controls.

\section{Data Synthesis and Analysis}

The impact of the COVID-19 pandemic on physical activity levels and sedentary behaviours was analysed by the narrative synthesis. Results were presented by grouping clinically similar studies. If convenient and sufficient data were available, it was performed narrative comparisons between the following groups: age, gender, disability group, and geographical area. A detailed analysis was conducted to examine the relationship between physical activity level and disease symptoms, and psychosocial factors. 


\section{Results}

\section{Search Results}

As shown in Fig. 1, 447 records initially were determined based on the search strategy. After deleting 44 duplicates, we removed 396 articles by reading their titles and abstracts. The full texts of the remaining seven articles were screened for eligibility. One article has been excluded because it was a case report. Six articles were included in this systematic review in the first systematic search on 22 September 2020. Eight more articles meeting the research criteria were added after the updated search on 4 February 2021.
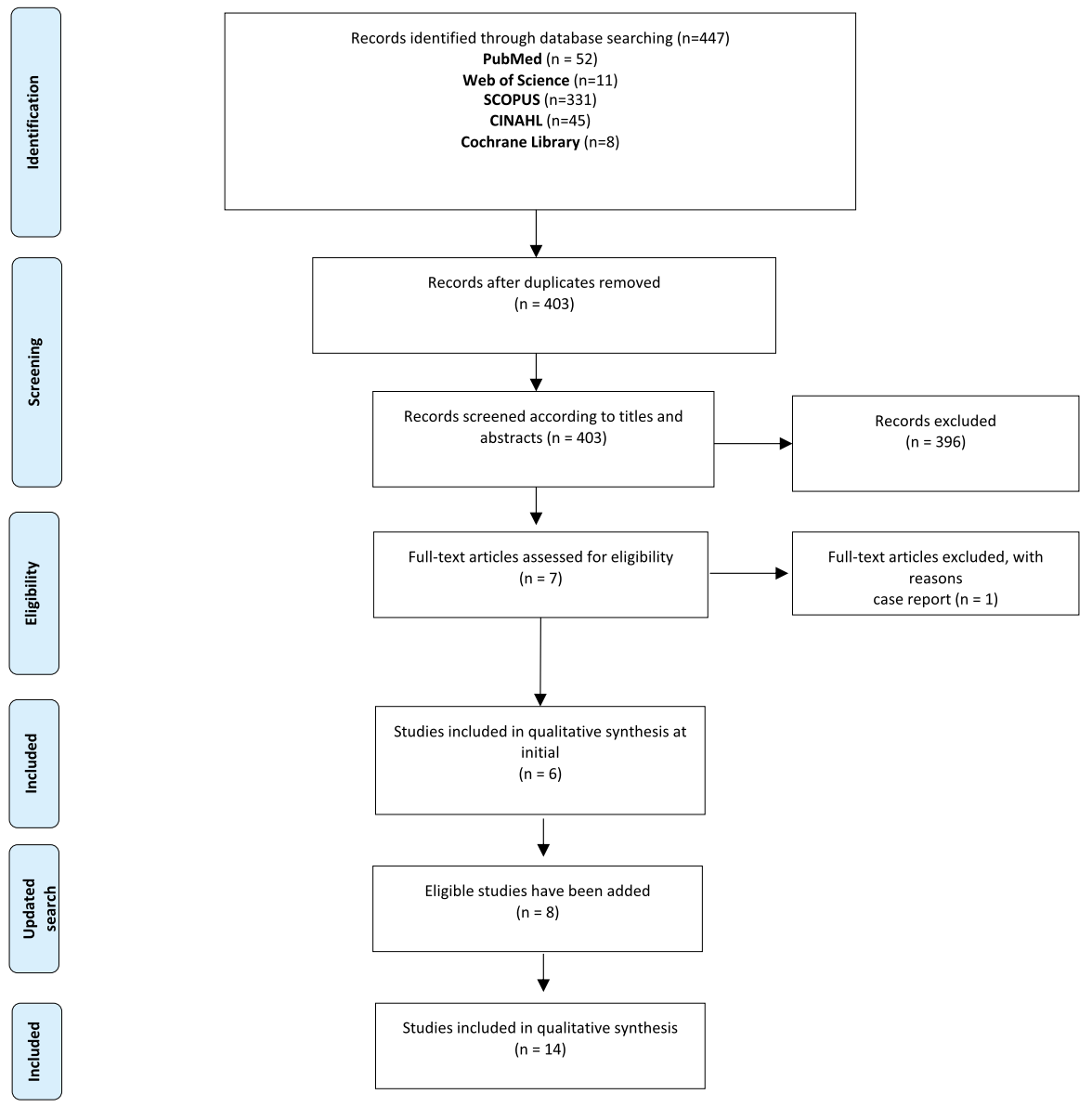

Fig. 1 PRISMA flow-chart 


\section{Study Characteristics}

Table 1 indicates the descriptive characteristics of the 14 included articles. Seven of the articles included persons with PD. The other studies $(n=7)$ enrolled participants with different pathologies including dementia $(n=1)$, multiple sclerosis $(n=1)$, spinal cord injury $(n=1)$, hereditary spastic paraplegia $(n=1)$, neuromuscular diseases $(n=1)$, Charcot-Marie-Tooth neuropathy $(n=1)$, and epilepsy $(n=1)$. Twelve studies questioned whether their physical activity and/or sports Questionnaires (IPAQ) and Physical Activity Scale for Individuals with Physical Disabilities (PASIPD) results assessed before and after the lockdown (Marco-Ahulló et al., 2021; Shalash et al., 2020) Three studies included healthy controls Brown et al., 2020; di Stefano et al., 2020; Shalash et al., 2020), but two of them compared physical activity levels of healthy controls and people with neurological diseases (di Stefano et al., 2020; Shalash et al., 2020). Two studies also included caregivers (Borges-Machado et al., 2020; Cavallieri et al., 2021) Answers were obtained from caregivers of dementia patients, whereas in the study that included persons with Parkinson's disease, the caregivers were asked when patients could not respond (Borges-Machado et al., 2020; Cavallieri et al., 2021). Brown et al. included participants with and without COVID-19 and presented subgroup analysis for the baseline characteristics, but they provided data for information on physical activity or exercise of participants without COVID-19 (Brown et al., 2020).

The overall methodological quality is presented in the Supplementary Table and Table 1. All the included studies met criteria 1, 2, 3, and 7 because they well explained research questions and study populations, achieved high participation rates of eligible persons, and sufficient timeframes for assessments. However, none of the studies met criteria 5, 6, 10, and 12. There was no sample size calculation, power description, follow-up, and blindness to the exposure status. assessment in any papers. Also, since the COVID-19 pandemic unexpectedly affects all participants of the studies, none of the studies could assess the exposures of interest before the outcomes were measured.

The date was not reported in the study conducted in Egypt, (Shalash et al., 2020 but the data collection was carried out in other studies between April and June when the measures were strict.

\section{Participants}

Fourteen studies included a total of 7662 persons with neurological diseases and 1663 healthy participants. Most of the studies included persons with PD $(90 \%$, $n=6898$ ). The mean age of the participants was 57.2 ranged from 42 to 74 years.

\section{Effects of the Pandemic on Physical Activity}

Three studies used the IPAQ to assess physical activity levels ${ }^{6,22,25}$. In the 11 studies, a constructed survey was used to determine whether physical activity/sport activities/ 


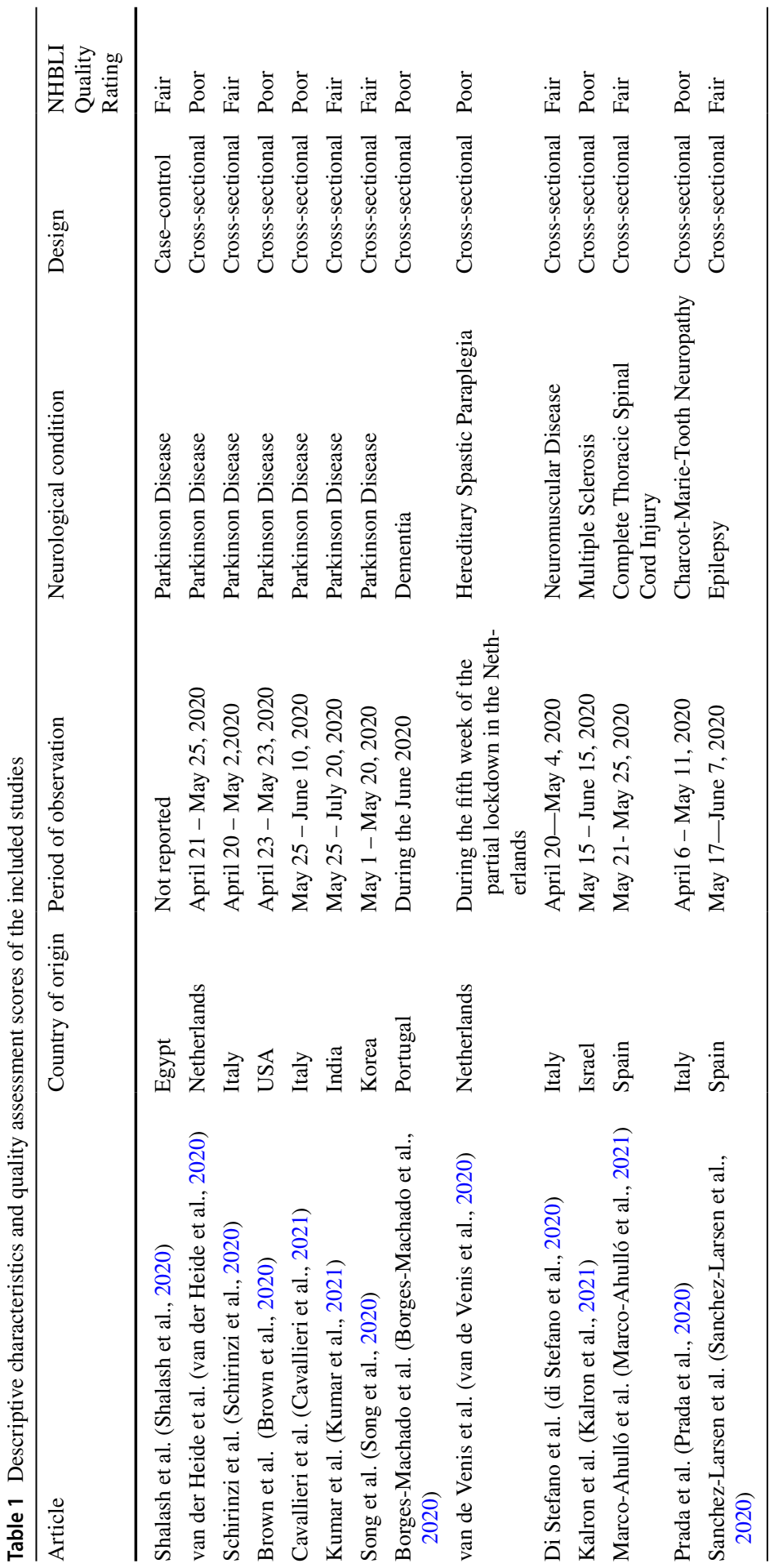


exercise has changed during the pandemic (Borges-Machado et al., 2020; Brown et al., 2020; Cavallieri et al., 2021; Kalron et al., 2021; Kumar et al., 2021; Prada et al., 2020; Sanchez-Larsen et al., 2020; Schirinzi et al., 2020; Song et al., 2020; van de Venis et al., 2020; van der Heide et al., 2020); (Schirinzi et al., 2020). used both IPAQ and structured surveys. Ahullo et al. applied PASIPD to manual wheelchair users with spinal cord injury (Marco-Ahulló et al., 2021).

Thirteen of the studies that evaluated physical activity changes reported a reduction in physical activity level following the COVID-19 pandemic. (Borges-Machado et al., 2020; Brown et al., 2020; Cavallieri et al., 2021; di Stefano et al., 2020; Kalron et al., 2021; Kumar et al., 2021; Marco-Ahulló et al., 2021; Prada et al., 2020; SanchezLarsen et al., 2020; Shalash et al., 2020; Song et al., 2020; van de Venis et al., 2020; van der Heide et al., 2020). Schrinzi et al. (Schirinzi et al., 2020) reported that the total number of patients playing sports was maintained but, during the pandemic, the total energy expenditure was 1994.7 \pm 1971 metabolic equivalent (MET) min/week in persons with PD, and this score was the leading risk factor for perceived clinical worsening. Besides, the number of patients under physiotherapy/rehabilitation was decreased by $78 \%$ in their study (Schirinzi et al., 2020). In Cavallieri et al.'s study, 52.24\% of the patients reported that the absence of physical activity is an unmet need during the pandemic (Cavallieri et al., 2021).

Across the studies that compared physical activity in persons with neurological diseases and healthy controls, both reported a greater physical activity reduction following the COVID-19 pandemic in persons with PD and neuromuscular disease (NMD) (di Stefano et al., 2020; Shalash et al., 2020). Brown et al. (Brown et al., 2020). compared only baseline characteristics, not the physical activity levels.

\section{Relationship Between Physical Activity and Disease Symptoms, and Psychosocial Factors During the Pandemic}

Of the studies involving persons with PD, Shalash et al. (Shalash et al., 2020). found that physical activity level is associated with depression, anxiety, pre-lockdown depression, and cognition, while van der Heide et al. (van der Heide et al., 2020). did not find a relationship between time of physical activity and perceived stress. The total MET score has been identified as the main risk factor for perceived deterioration of global health by Schirinzi et al. (Schirinzi et al., 2020). Heide et al. found that reduction of physical activity correlated with worsening of symptoms including rigidity, tremor, pain, and fatigue (van der Heide et al., 2020). Similarly, Brown et al. (Brown et al., 2020). reported that cancelled or postponed exercise activities increase the risk of the worsening of motor, cognitive, mood, autonomic, and sleep symptoms and Song et al. indicated that a decrease in the amount of exercise associated with worsening in motor and non-motor symptoms of Parkinson disease (Song et al., 2020). A decrease in physical activity and an increase in the number of patients with screen time of more than $3 \mathrm{~h}$ were also found to be associated with new-onset sleep disorders (Kumar et al., 2021).

The physical and mental components of the 12-Item Short Form Survey (SF-12) were correlated with a decrease in physical activity levels in persons with NMD (di 
Stefano et al., 2020). van de Venis et al. (van de Venis et al., 2020). showed that persons with hereditary spastic paraplegia (HSP) with a reduced level of physical activity experienced increased disease symptoms like increased muscle stiffness, pain, physical fatigue, and gait impairments. Also, it has been reported that a decrease in physical activity is associated with worsening of seizures in individuals with epilepsy (Sanchez-Larsen et al., 2020) (Table 2).

\section{Discussion}

This paper is the first systematic review to reveal the change of physical activity level in people with neurological diseases during the COVID-19 pandemic period. The evidence presented in this review highlights the ongoing COVID-19 pandemic affects the physical activity levels of people with neurological disease. Studies recruiting 7662 persons with neurological disease and 1663 healthy controls indicate that physical activity level decreased in the period of the COVID-19 pandemic. Additionally, the reduction of physical activity was related to depression, perceived health, the mental and physical component of quality of life, and worse disease symptoms.

The sudden radical change in the lifestyle may lead to an increase in sedentary behaviour and a decrease in the physical activity level in the period of the COVID-19 pandemic. However, regular physical activity is required to counteract the negative effects of diseases in persons with neurological diseases (Block et al., 2016; Ellis \& Motl, 2013). The unintended decrease in daily physical activity and increased sedentary time can culminate changes in skeletal muscles, including loss of muscle strength, muscle power, and muscle mass associated with disuse and deterioration in cardiovascular health in after a short period of inactivity (Pagano et al., 2018; Pecanha et al., 2020). These changes may prepare the ground for further motor deterioration caused by deconditioning in persons with neurological diseases who already have motor impairments. Additionally, the preventive role of exercise for COVID-19 and its helping manage recovery from the COVID-19 through the mechanism, including enhanced metabolic homeostasis, suppress inflammation, and improvements in cardio-respiratory fitness, have been discussed in the literature (M. Wang, Baker, et al., 2020; Wang, Chao, et al., 2020). Considering these factors, the decrease in physical activity in individuals with neurological diseases indicates a problem that requires an urgent solution. Although supervised physiotherapy interventions are generally prescribed for persons with neurological disease, the hospitals and external centres had to reduce/stop their outpatient activities. In the study of Schirinzi et al. (Schirinzi et al., 2020), 78\% of physiotherapy sessions were interrupted in persons with PD. Although the effects of these interruptions are not known yet, they should be examined in future longitudinal studies.

There is evidence on that physical activity is not only related to physical health but also mental health through the brain and systemic effects in neurological diseases (Dauwan et al., 2019). It has been reported that neuropsychiatric sequel is caused by the immunological responses of the SARS-CoV-2 in the central nervous system and the psychological distress caused by a global pandemic (Troyer et al., 2020). 


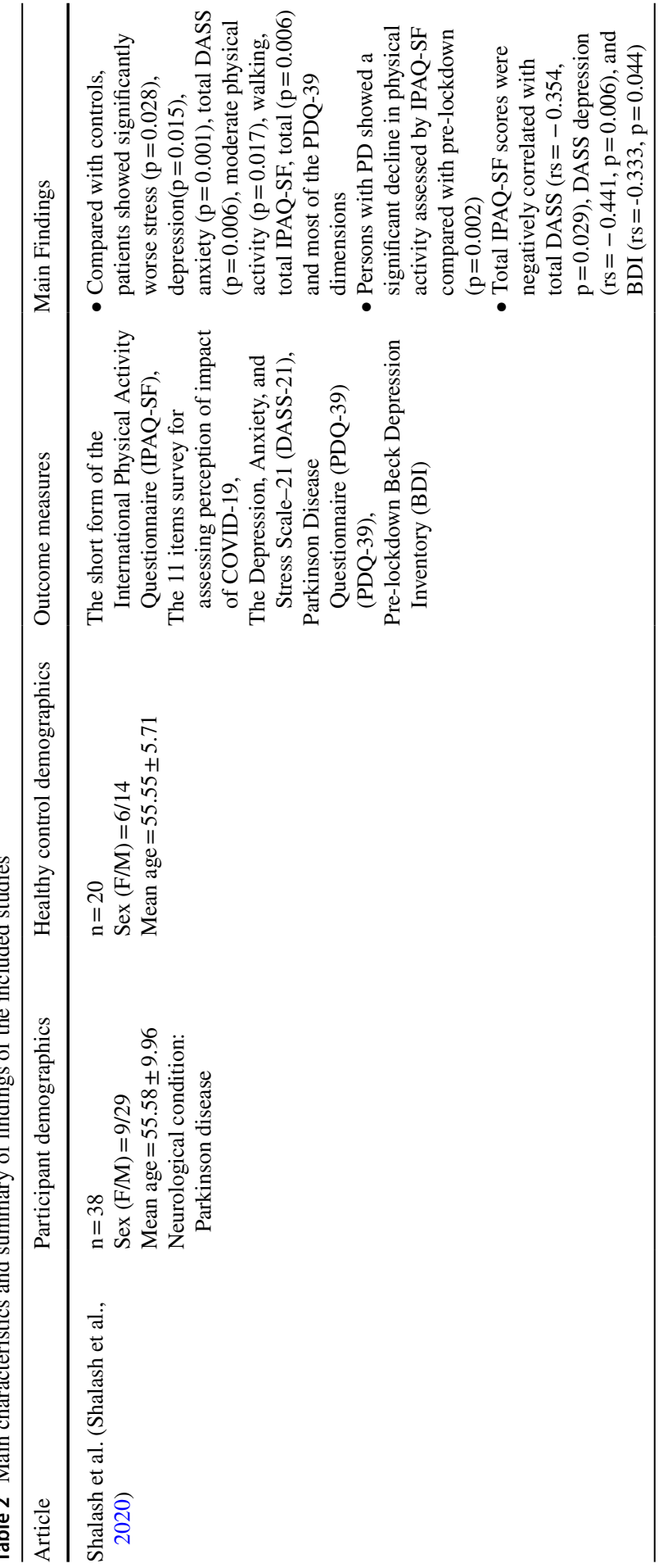




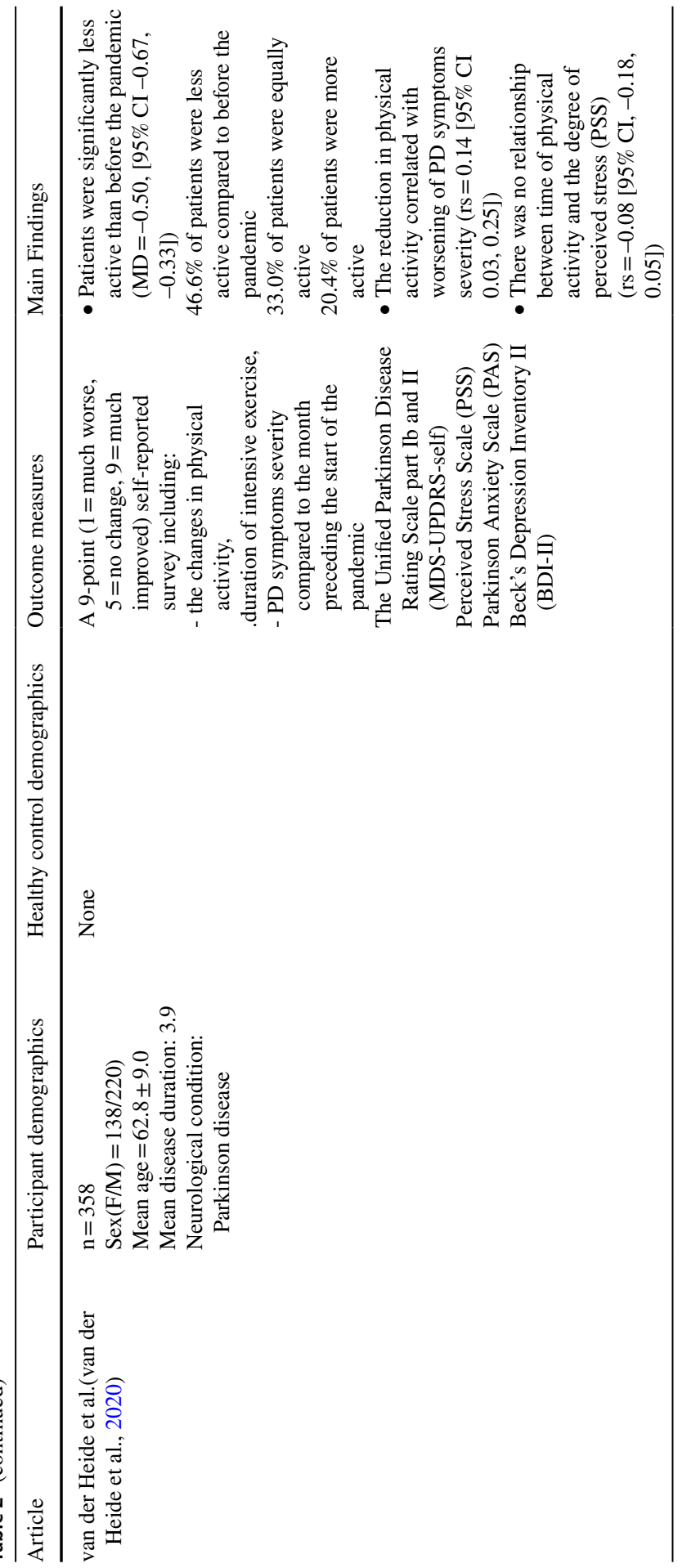




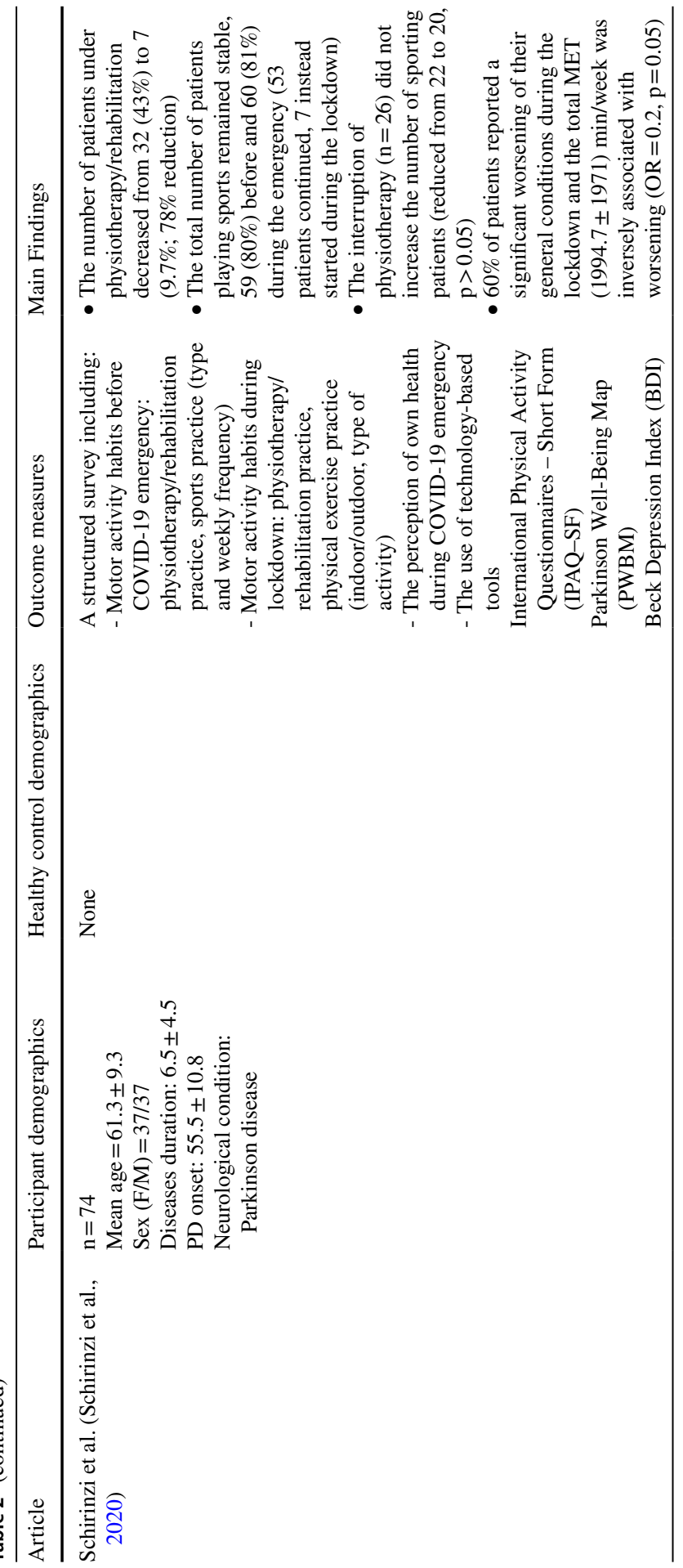




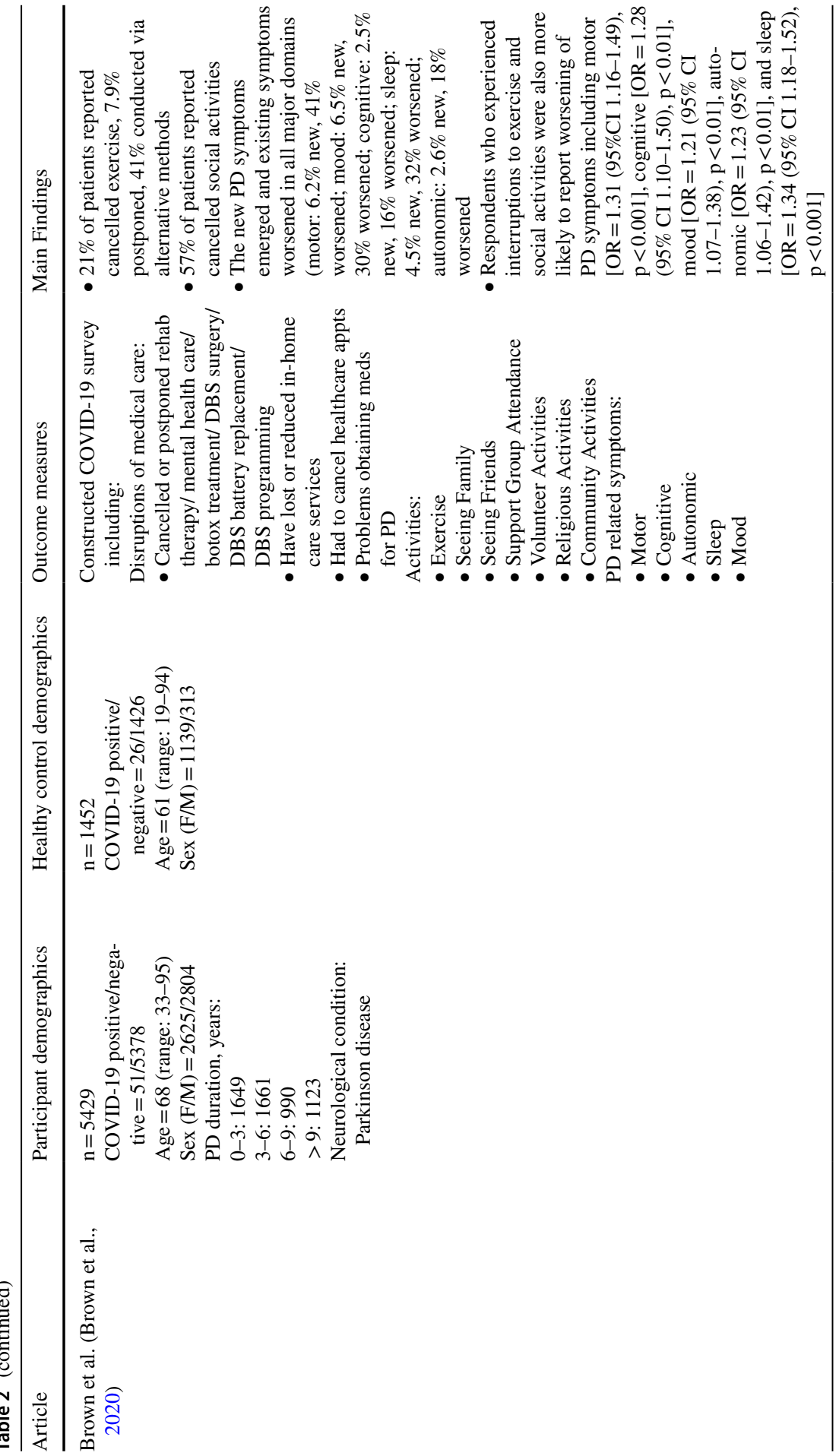




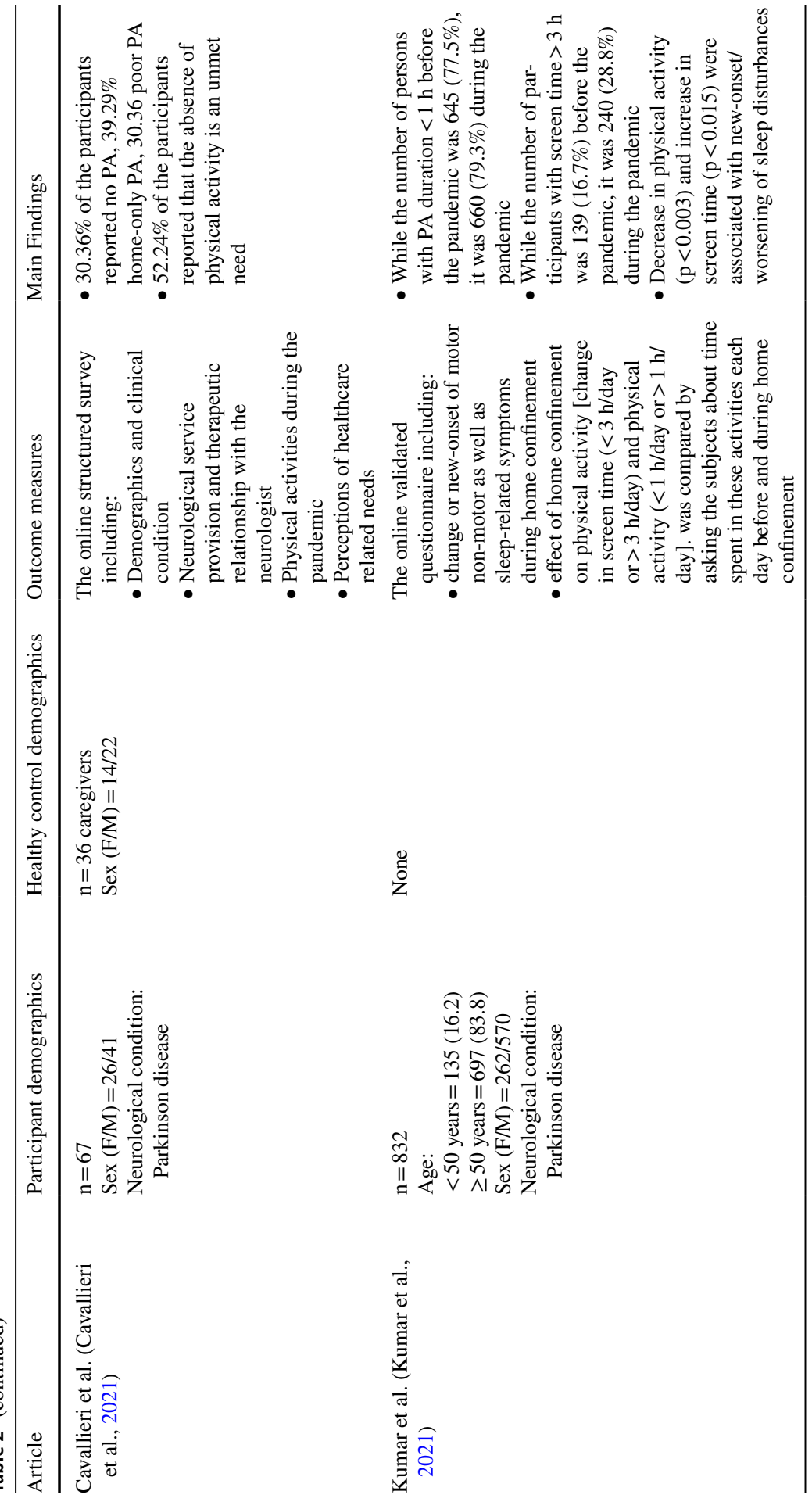




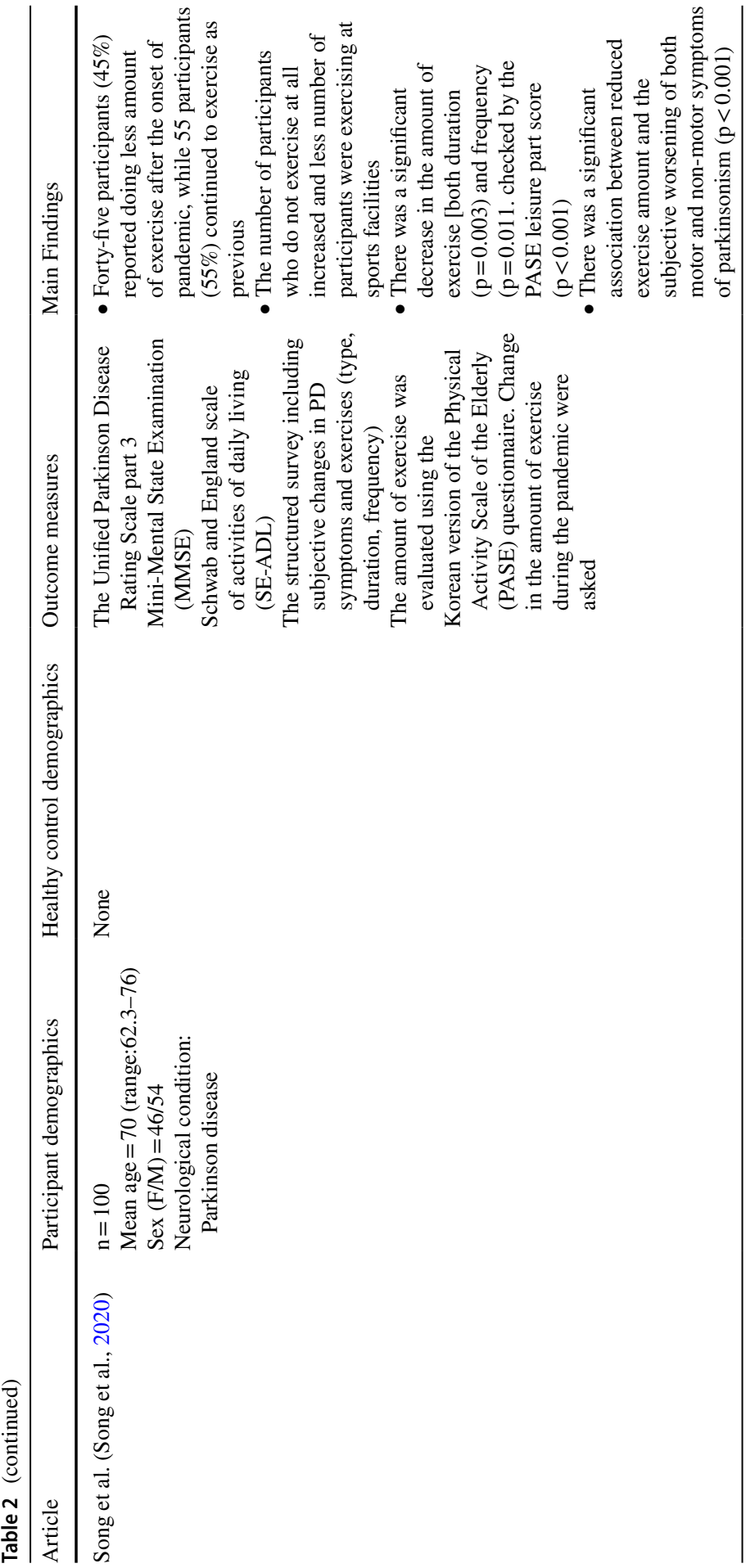




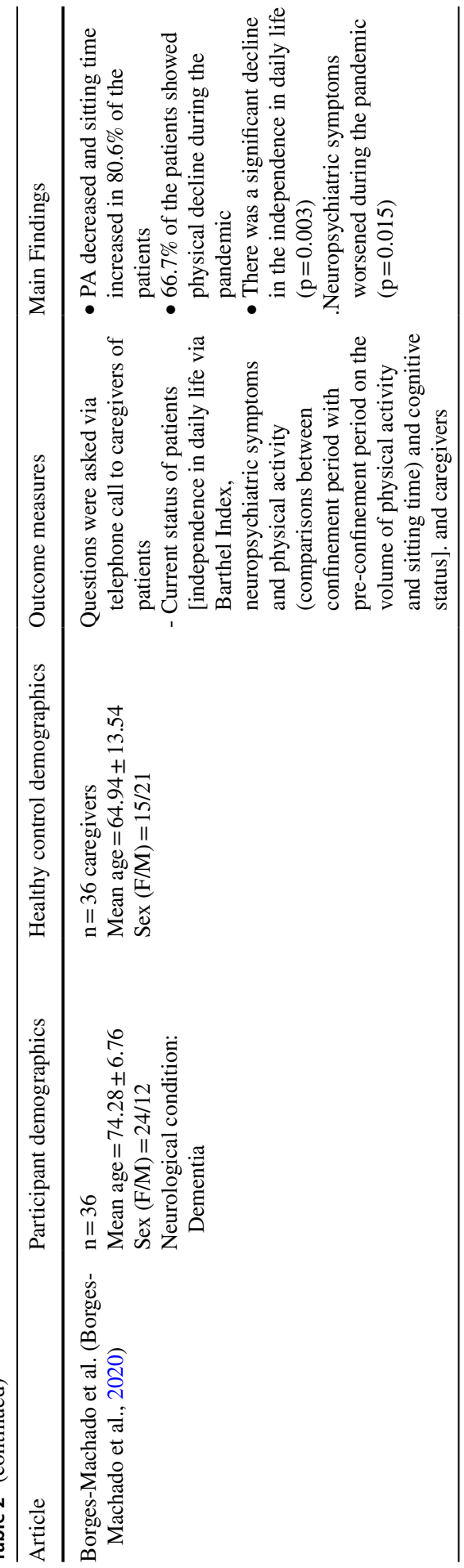




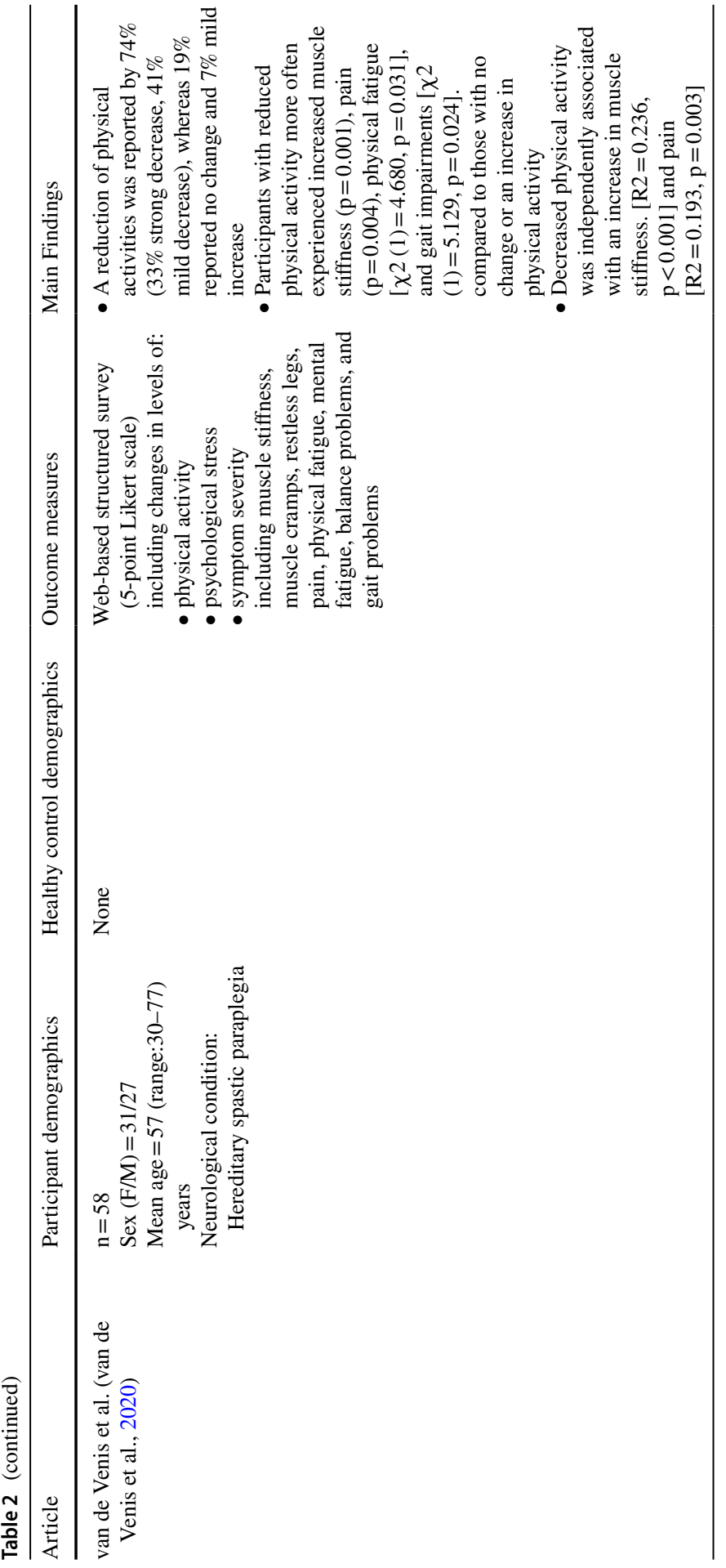




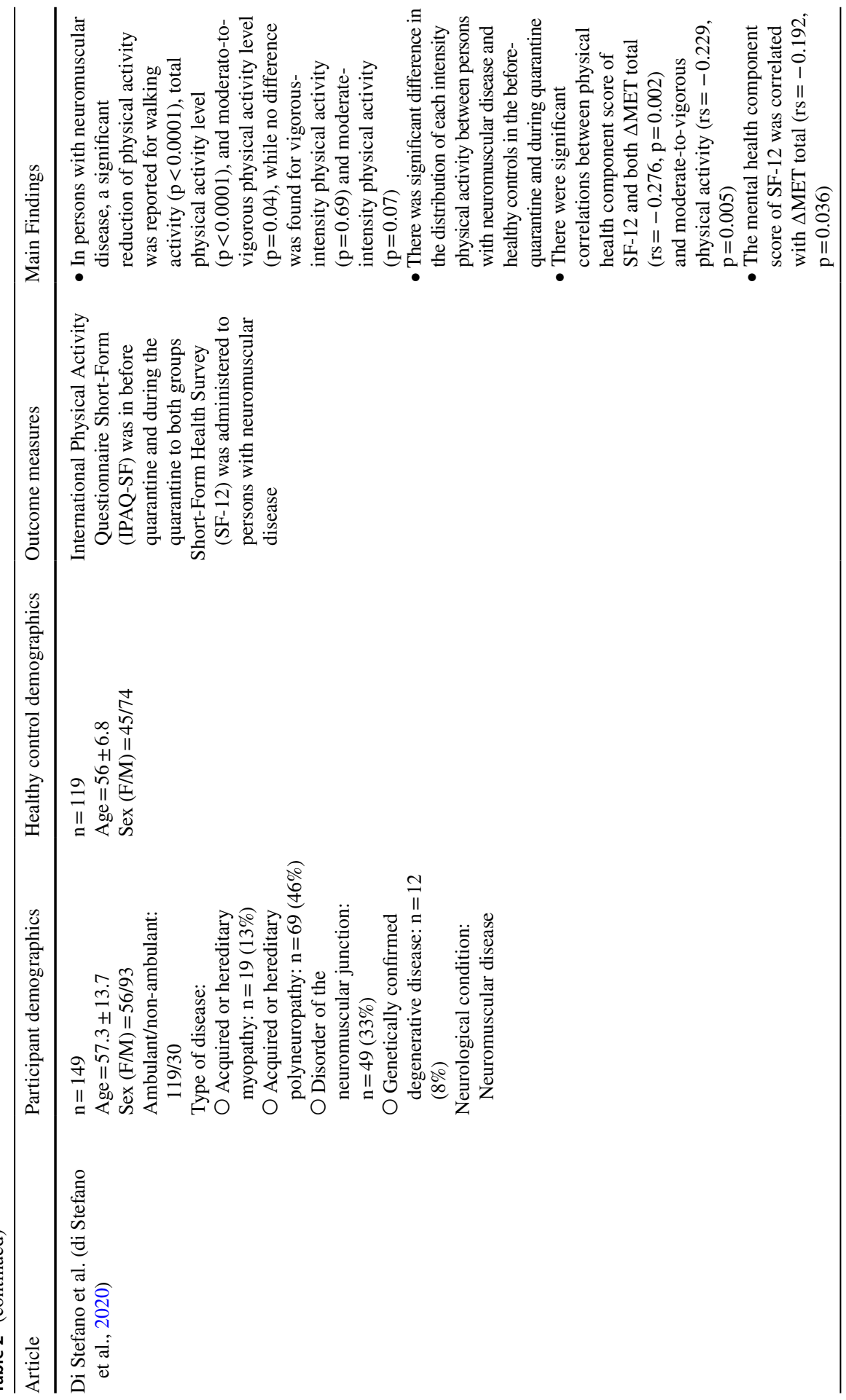




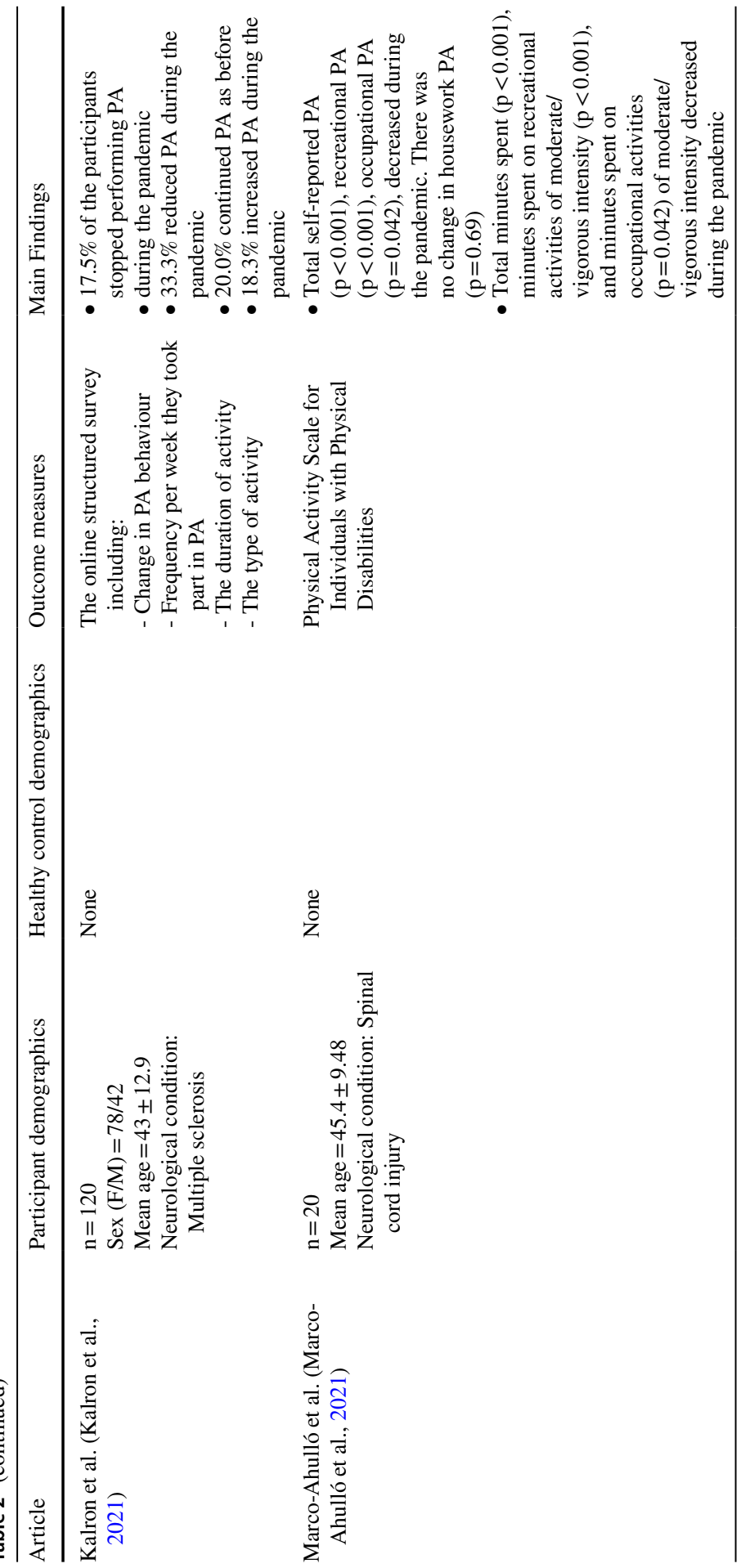



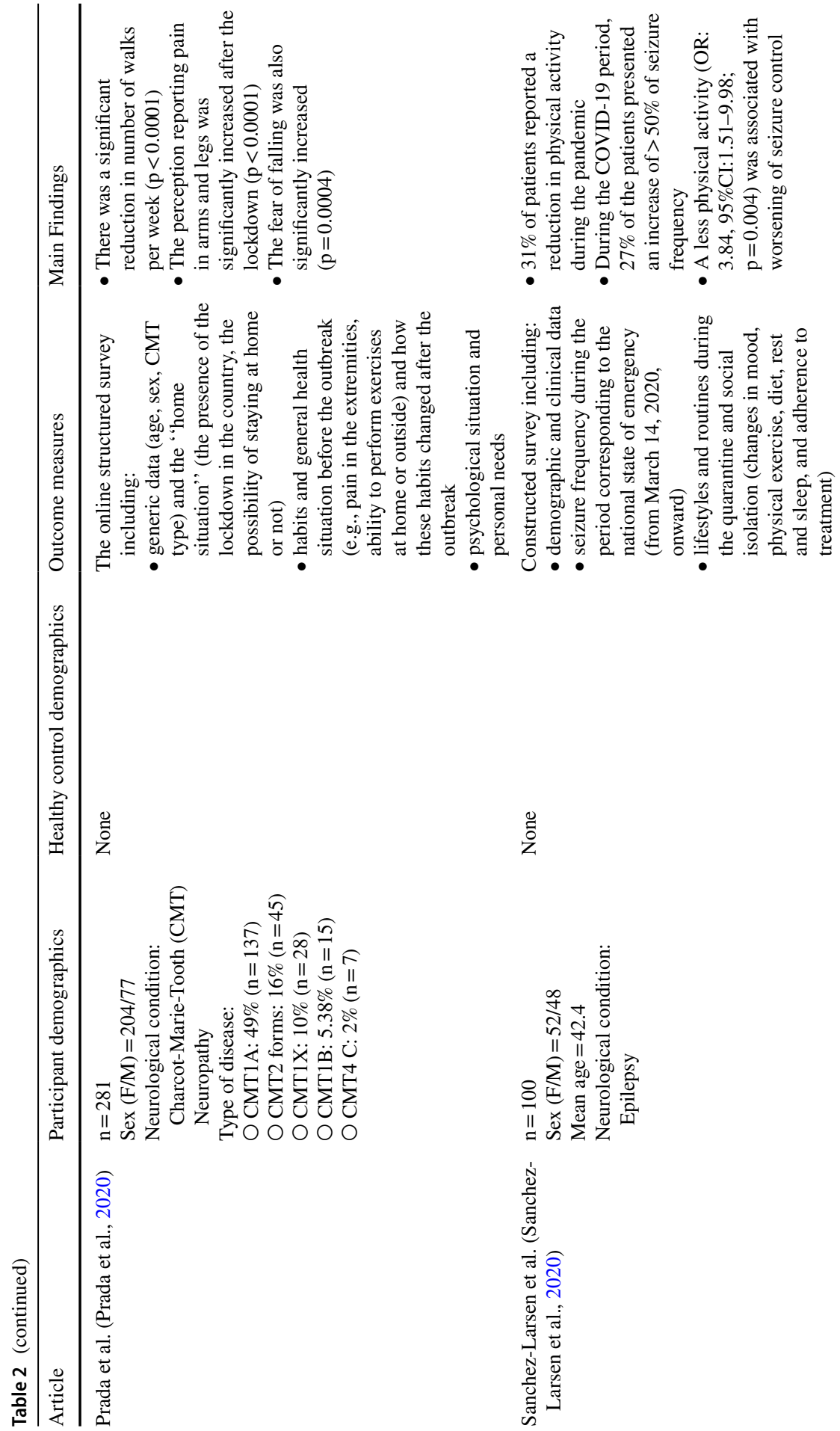

ถี

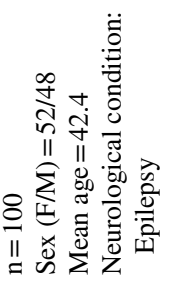

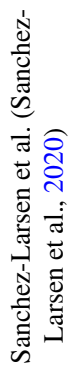


Therefore, maintaining physical activity shows an important role in this period. There is growing evidence that physical activity and exercise positively affect anxiety and stress through their neurobiological effects (McDowell et al., 2019; Mochcovitch et al., 2016; Morgan et al., 2015). In line with this mechanism, three studies in this review demonstrated that physical activity is related to depression, anxiety, perceived global health, and mental and physical dimensions quality of life in persons with PD and NMD (di Stefano et al., 2020; Schirinzi et al., 2020; Shalash et al., 2020). However, in the study of van der Heide, (van der Heide et al., 2020). time of physical activity and perceived stress were not correlated in persons with PD. These conflicting results may result from using different measurement tools and statistical methods of the included papers.

It has been well-known that individuals with chronic neurological conditions need regular rehabilitation, and adequate physical activity is critical for their health status (Abbruzzese et al., 2016; Belagaje, 2017; Block et al., 2016; Charytoniuk et al., 2020; Ellis \& Motl, 2013; Learmonth \& Motl, 2016). In the literature, some reports state that neurorehabilitation should not be delayed or interrupted during the pandemic period, which will shape the lifestyle until the treatment becomes available (Leocani et al., 2020; Manto et al., 2020; Mantovani et al., 2020; C. C. Wang, Chao, et al., 2020; Wang, Baker, et al., 2020). However, the reorganization of the health system worldwide due to the COVID 19 pandemic deeply affected the patients' in-person rehabilitation session (Guidon \& Amato, 2020; Mauri et al., 2020). When considering the close interaction between therapist and patient, it is not astounding that one of the most interrupted health services is rehabilitation sessions in the pandemic era (Boldrinı et al., 2020). For this reason, the accessibility of technology-based approaches such as telehealth delivery can be promoted to monitor remotely and increase the level of physical activity during pandemic conditions. To continue the rehabilitation sessions remotely, methods such as sending videos and photographs, performing online home visits were used (Manto et al., 2020; Mantovani et al., 2020). However, it is not yet clear to what extent patients have access to these opportunities and how effective they find these methods.

Our study has some limitations. Firstly, in the vast majority of studies, physical activity change was questioned cross-sectionally with patient-reported outcomes, and confounders were not controlled except four studies (Brown et al., 2020; Kumar et al., 2021; Schirinzi et al., 2020; Song et al., 2020). Also, while a majority of the studies did not assess the sitting time, which could be important for sedentary behaviour. In some studies, the IPAQ was used, but the sitting sub-score has not been provided (di Stefano et al., 2020; Schirinzi et al., 2020; Shalash et al., 2020). Physical activity level was not objectively monitored using smart technologies in any study. Pre-pandemic physical activity level was known only in three studies (Shalash et al., 2020). Besides, in some studies, the lack of a control group may hinder understanding whether the results are disease specific. Lastly, although all studies present results in the same direction, the fact that the majority of the participants are persons with PD makes the generalizability of the results difficult.

An issue that attracts attention in the current systematic review was 7 of the 14 studies included in the research have poor quality (Borges-Machado et al., 2020; Brown et al., 2020; Cavallieri et al., 2021; Kalron et al., 2021; Prada et al., 2020; 
van de Venis et al., 2020; van der Heide et al., 2020). Although it can affect the generalizability of the results, it is understandable that the studies are planned and conducted quickly due to the pandemic's sudden nature. However, it is believed to be important that combining the results of existing research in line with a consociate conclusion until studies with high-quality study design offering more important evidence are published will make significant contributions to the literature.

There is a need for longitudinal studies that will examine the long-term effects of a reduction in physical activity during the quarantine period and examine the level of physical activity after progressive return to everyday life. However, these days some countries face the new mutation of the virus and an increase in the number of cases, which could lead to new challenges. Therefore, there is a need for urgent action to maintain the physical activity level. Since the end of the pandemic cannot be predicted, prevention strategies should be designed against these rapid changes in physical activity. Future studies should investigate the effects of interventions or alternative ways targeting physical activity during the pandemic.

\section{Conclusions}

This review highlights the negative indirect impact of the COVID-19 pandemic on physical activity levels in persons with neurological diseases. Moreover, the reduction of physical activity was associated with depression, perceived health, the mental and physical component of quality of life, and worse disease symptoms. Since the continuing spread of the pandemic and the associated global disruption, rapid modifications to increase physical activity should be sought within the measures' framework.

Supplementary Information The online version contains supplementary material available at https://oi. org/10.1007/s10882-022-09836-X.

Authors' Contributions Conceptualization: ZA, MK, TK; Methodology: ZA, MK, TK; Data curation: ZA, MK; Supervision: TK; Visualization: ZA, MK, TK; Writing-original draft: ZA, MK; Writing review \& editing: ZA, MK, TK.

Authors read and approved the final version of the manuscript; all authors indicated in the byline meet the criteria for authorship.

Funding This research did not receive any specific grant from funding agencies in the public, commercial, or not-for-profit sectors.

Data Availability The data of the study was not stored in a public database. However, in a necessary situation, data will be shared if data is requested from the author.

Code Availability Not applicable.

\section{Declarations}

Conflicts of Interest Authors have nothing to disclose. 


\section{References}

Abbruzzese, G., Marchese, R., Avanzino, L., \& Pelosin, E. (2016). Rehabilitation for Parkinson's disease: Current outlook and future challenges. Parkinsonism and Related Disorders, 22, S60-S64. https://doi.org/10.1016/j.parkreldis.2015.09.005

ACSM. (2020). Staying active during the Coronavirus pandemic. Exercise is Medicine.

Amekran, Y., \& el Hangouche, A. J. (2021). Coronavirus disease (COVID-19) and the need to maintain regular physical activity. The Journal of sports medicine and physical fitness. https://doi.org/ 10.23736/S0022-4707.20.11524-X

Belagaje, S. R. (2017). Stroke Rehabilitation. CONTINUUM Lifelong Learning in Neurology. https:// doi.org/10.1212/CON.0000000000000423

Block, V. A. J., Pitsch, E., Tahir, P., Cree, B. A. C., Allen, D. D., \& Gelfand, J. M. (2016). Remote physical activity monitoring in neurological disease: A systematic review. PLoS ONE. https://doi. org/10.1371/journal.pone.0154335

Boldrini, P., Bernetti, A., \& Fiore, P. (2020). Impact of COVID-19 outbreak on rehabilitation services and Physical and Rehabilitation Medicine physicians' activities in Italy An official document of the Italian PRM Society (SIMFER). European Journal of Physical and Rehabilitation Medicine. https:// doi.org/10.23736/S1973-9087.20.06256-5

Borges-Machado, F., Barros, D., Ribeiro, Ó., \& Carvalho, J. (2020). The Effects of COVID-19 Home Confinement in Dementia Care: Physical and Cognitive Decline, Severe Neuropsychiatric Symptoms and Increased Caregiving Burden. American journal of Alzheimer's disease and other dementias, 35.https://doi.org/10.1177/1533317520976720

Boysen, G., \& Krarup, L. H. (2009). Benefits of physical activity for stroke survivors. Expert Review of Neurotherapeutics. https://doi.org/10.1586/14737175.9.2.147

Brown, E. G., Chahine, L. M., Goldman, S. M., Korell, M., Mann, E., Kinel, D. R., et al. (2020). The Effect of the COVID-19 Pandemic on People with Parkinson's Disease. Journal of Parkinson's Disease, 10(4), 1365-1377. https://doi.org/10.3233/JPD-202249

Cavallieri, F., Sireci, F., Fioravanti, V., Toschi, G., Rispoli, V., Antonelli, F., et al. (2021). Parkinson Patients' needs during COVID-19 pandemic in a red zone: A Framework Analysis of OpenEnded Survey Questions. European Journal of Neurology. https://doi.org/10.1111/ene.14745

Charytoniuk, T., Zywno, H., Konstantynowicz-Nowicka, K., Berk, K., Bzdega, W., \& Chabowski, A. (2020). Can physical activity support the endocannabinoid system in the preventive and therapeutic approach to neurological disorders? International Journal of Molecular Sciences. https:// doi.org/10.3390/ijms21124221

Dauwan, M., Begemann, M. J. H., Slot, M. I. E., Lee, E. H. M., Scheltens, P., \& Sommer, I. E. C. (2019). Physical exercise improves quality of life, depressive symptoms, and cognition across chronic brain disorders: a transdiagnostic systematic review and meta-analysis of randomized controlled trials. Journal of Neurology, 1-25.https://doi.org/10.1007/s00415-019-09493-9

di Stefano, V., Battaglia, G., Giustino, V., Gagliardo, A., D’Aleo, M., Giannini, O., et al. (2020). Significant reduction of physical activity in patients with neuromuscular disease during COVID-19 pandemic: the long-term consequences of quarantine. Journal of Neurology, 1-7.https://doi.org/ 10.1007/s00415-020-10064-6

Ellis, T., \& Motl, R. W. (2013). Physical activity behavior change in persons with neurologic disorders: Overview and examples from Parkinson disease and multiple sclerosis. Journal of Neurologic Physical Therapy. https://doi.org/10.1097/NPT.0b013e31829157c0

Elsworth, C., Dawes, H., Sackley, C., Soundy, A., Howells, K., Wade, D., et al. (2009). A study of perceived facilitators to physical activity in neurological conditions. International Journal of Therapy and Rehabilitation, 16(1), 17-23. https://doi.org/10.12968/ijtr.2009.16.1.37936

Ferini-Strambi, L., \& Salsone, M. (2020). COVID-19 and neurological disorders: are neurodegenerative or neuroimmunological diseases more vulnerable? Journal of Neurology, 1-11.https://doi. org/10.1007/s00415-020-10070-8

Goodwin, V. A., Richards, S. H., Taylor, R. S., Taylor, A. H., \& Campbell, J. L. (2008). The effectiveness of exercise interventions for people with Parkinson's disease: A systematic review and metaanalysis. Movement Disorders. https://doi.org/10.1002/mds.21922

Guidon, A. C., \& Amato, A. A. (2020). COVID-19 and neuromuscular disorders. Neurology. https:// doi.org/10.1212/WNL.0000000000009566 
Hammami, A., Harrabi, B., Mohr, M., \& Krustrup, P. (2020). Physical activity and coronavirus disease 2019 (COVID-19): Specific recommendations for home-based physical training. Managing Sport and Leisure. https://doi.org/10.1080/23750472.2020.1757494

Jiménez-Pavón, D., Carbonell-Baeza, A., \& Lavie, C. J. (2020). Physical exercise as therapy to fight against the mental and physical consequences of COVID-19 quarantine: Special focus in older people. Progress in Cardiovascular Diseases. https://doi.org/10.1016/j.pcad.2020.03.009

Kalron, A., Dolev, M., Greenberg-Abrahami, M., Menascu, S., Frid, L., Avrech-Shezifi, S., et al. (2021). Physical activity behavior in people with multiple sclerosis during the COVID-19 pandemic in Israel: Results of an online survey. Multiple Sclerosis and Related Disorders, 47. https://doi.org/10.1016/j. msard.2020.102603

Kumar, N., Gupta, R., Kumar, H., Mehta, S., Rajan, R., Kumar, D., et al. (2021). Impact of home confinement during COVID-19 pandemic on sleep parameters in Parkinson's disease. Sleep Medicine, 77, 15-22. https://doi.org/10.1016/j.sleep.2020.11.021

Learmonth, Y. C., \& Motl, R. W. (2016). Physical activity and exercise training in multiple sclerosis: A review and content analysis of qualitative research identifying perceived determinants and consequences. Disability and Rehabilitation. https://doi.org/10.3109/09638288.2015.1077397

Leocani, L., Diserens, K., Moccia, M., \& Caltagirone, C. (2020). Disability through COVID-19 pandemic: Neurorehabilitation cannot wait. European Journal of Neurology. https://doi.org/10.1111/ene.14320

Manto, M., Dupre, N., Hadjivassiliou, M., Louis, E. D., Mitoma, H., Molinari, M., et al. (2020). Management of Patients with Cerebellar Ataxia During the COVID-19 Pandemic: Current Concerns and Future Implications. Cerebellum, 19(4), 562-568. https://doi.org/10.1007/s12311-020-01139-1

Mantovani, E., Zucchella, C., Bottiroli, S., Federico, A., Giugno, R., Sandrini, G., et al. (2020). Telemedicine and Virtual Reality for Cognitive Rehabilitation: A Roadmap for the COVID-19 Pandemic. Frontiers in Neurology, 11, 926. https://doi.org/10.3389/fneur.2020.00926

Marco-Ahulló, A., Montesinos-Magraner, L., González, L.-M., Morales, J., Bernabéu-García, J. A., \& García-Massó, X. (2021). Impact of COVID-19 on the self-reported physical activity of people with complete thoracic spinal cord injury full-time manual wheelchair users. The Journal of Spinal Cord Medicine, 1-5. https://doi.org/10.1080/10790268.2020.1857490

Mauri, E., Abati, E., Musumeci, O., Rodolico, C., D’Angelo, M. G., Mirabella, M., et al. (2020). Estimating the impact of COVID-19 pandemic on services provided by Italian Neuromuscular Centers: an Italian Association of Myology survey of the acute phase. Acta Myologica, 39(2), 57-66. https://doi.org/10. 36185/2532-1900-008

McDowell, C. P., Dishman, R. K., Gordon, B. R., \& Herring, M. P. (2019). Physical Activity and Anxiety: A Systematic Review and Meta-analysis of Prospective Cohort Studies. American Journal of Preventive Medicine. https://doi.org/10.1016/j.amepre.2019.05.012

Mochcovitch, M. D., Deslandes, A. C., Freire, R. C., Garcia, R. F., \& Nardi, A. E. (2016). The effects of regular physical activity on anxiety symptoms in healthy older adults: A systematic review. Revista Brasileira De Psiquiatria. https://doi.org/10.1590/1516-4446-2015-1893

Moher, D., Liberati, A., Tetzlaff, J., Altman, D. G., Altman, D., Antes, G., et al. (2009). Preferred reporting items for systematic reviews and meta-analyses: The PRISMA statement. PLoS Medicine. https://doi. org/10.1371/journal.pmed.1000097

Morgan, J. A., Corrigan, F., \& Baune, B. T. (2015). Effects of physical exercise on central nervous system functions: A review of brain region specific adaptations. Journal of Molecular Psychiatry, 3(1), 3. https://doi. org/10.1186/s40303-015-0010-8

NHLBI. (2014). Quality Assessment Tool for Observational Cohort and Cross-Sectional Studies. Bethesda, MD: National Institutes of Health, Department of Health and Human Services., 1-4. https://www.nhlbi. nih.gov/health-pro/guidelines/in-develop/cardiovascular-risk-reduction/tools/cohort

Pagano, A. F., Brioche, T., Arc-Chagnaud, C., Demangel, R., Chopard, A., \& Py, G. (2018). Short-term disuse promotes fatty acid infiltration into skeletal muscle. Journal of Cachexia, Sarcopenia and Muscle, 9(2), 335-347. https://doi.org/10.1002/jcsm.12259

Pecanha, T., Goessler, K. F., Roschel, H., \& Gualano, B. (2020). Social isolation during the COVID-19 pandemic can increase physical inactivity and the global burden of cardiovascular disease. American Journal of Physiology - Heart and Circulatory Physiology. https://doi.org/10.1152/ajpheart.00268.2020

Prada, V., Hamedani, M., Genovese, F., Zuppa, A., Benedetti, L., Bellone, E., et al. (2020). People with Charcot-Marie-Tooth disease and COVID-19: Impaired physical conditions due to the lockdown. An International cross-sectional survey. Annals of Physical and Rehabilitation Medicine. https://doi.org/10. 1016/j.rehab.2020.10.001 
Quinn, L., MacPherson, C., Long, K., \& Shah, H. (2020). Promoting physical activity via telehealth in people with parkinson disease: The path forward after the COVID-19 pandemic? Physical Therapy, 100(10), 1730-1736. https://doi.org/10.1093/ptj/pzaa128

Reynolds, G. (2020, January 4). Stuck Inside? Keep Walking. The New York Times.

Sanchez-Larsen, A., Gonzalez-Villar, E., Díaz-Maroto, I., Layos-Romero, A., Martínez-Martín, Á., AlcahutRodriguez, C., et al. (2020). Influence of the COVID-19 outbreak in people with epilepsy: Analysis of a Spanish population (EPICOVID registry). Epilepsy and Behavior, 112. https://doi.org/10.1016/j.yebeh. 2020.107396

Santos, C. M. D. C., Pimenta, C. A. D. M., \& Nobre, M. R. C. (2007). A estratégia PICO para a construção da pergunta de pesquisa e busca de evidências. Revista Latino-Americana De Enfermagem, 15(3), 508511. https://doi.org/10.1590/S0104-11692007000300023

Schirinzi, T., di Lazzaro, G., Salimei, C., Cerroni, R., Liguori, C., Scalise, S., et al. (2020). Physical Activity Changes and Correlate Effects in Patients with Parkinson's Disease during COVID-19 Lockdown. Movement Disorders Clinical Practice, 7(7), 797-802. https://doi.org/10.1002/mdc3.13026

Shalash, A., Roushdy, T., Essam, M., Fathy, M., Dawood, N. L., Abushady, E. M., et al. (2020). Mental Health, Physical Activity, and Quality of Life in Parkinson's Disease During COVID-19 Pandemic. Movement Disorders. https://doi.org/10.1002/mds.28134

Song, J., Ahn, J. H., Choi, I., Mun, J. K., Cho, J. W., \& Youn, J. (2020). The changes of exercise pattern and clinical symptoms in patients with Parkinson's disease in the era of COVID-19 pandemic. Parkinsonism and Related Disorders, 80, 148-151. https://doi.org/10.1016/j.parkreldis.2020.09.034

Stennett, A., de Souza, L., \& Norris, M. (2020). The meaning of exercise and physical activity in community dwelling people with multiple sclerosis. Disability and Rehabilitation, 42(3), 317-323. https://doi.org/ 10.1080/09638288.2018.1497715

Stroud, N. M., \& Minahan, C. L. (2009). The impact of regular physical activity on fatigue, depression and quality of life in persons with multiple sclerosis. Health and Quality of Life Outcomes, 7(1), 68. https:// doi.org/10.1186/1477-7525-7-68

Tison, G. H., Avram, R., Kuhar, P., Abreau, S., Marcus, G. M., Pletcher, M. J., \& Olgin, J. E. (2020). Worldwide Effect of COVID-19 on Physical Activity: A Descriptive Study. Annals of Internal Medicine. https://doi.org/10.7326/M20-2665

Tremblay, M. S., Warburton, D. E. R., Janssen, I., Paterson, D. H., Latimer, A. E., Rhodes, R. E., et al. (2011). New Canadian physical activity guidelines. Applied Physiology, Nutrition and Metabolism. https://doi.org/10.1139/H11-009

Troyer, E. A., Kohn, J. N., \& Hong, S. (2020). Are we facing a crashing wave of neuropsychiatric sequelae of COVID-19? Neuropsychiatric symptoms and potential immunologic mechanisms. Brain, Behavior, and Immunity. https://doi.org/10.1016/j.bbi.2020.04.027

van de Venis, L., van de Warrenburg, B. P. C., Weerdesteyn, V., van Lith, B. J. H., Geurts, A. C. H., \& Nonnekes, J. (2020). COVID-19 reveals influence of physical activity on symptom severity in hereditary spastic paraplegia. Journal of Neurology. https://doi.org/10.1007/s00415-020-10016-0

van der Heide, A., Meinders, M. J., Bloem, B. R., \& Helmich, R. C. (2020). The Impact of the COVID-19 Pandemic on Psychological Distress, Physical Activity, and Symptom Severity in Parkinson's Disease. Journal of Parkinson's Disease. https://doi.org/10.3233/JPD-202251

van Tulder, M., Furlan, A., Bombardier, C., \& Bouter, L. (2003). Updated method guidelines for systematic reviews in the Cochrane Collaboration Back Review Group. Spine. https://doi.org/10.1097/00007632200306150-00014

Wang, C. C., Chao, J. K., Wang, M. L., Yang, Y. P., Chien, C. S., Lai, W. Y., et al. (2020). Care for Patients with Stroke During the COVID-19 Pandemic: Physical Therapy and Rehabilitation Suggestions for Preventing Secondary Stroke. Journal of Stroke and Cerebrovascular Diseases. https://doi.org/10.1016/j. jstrokecerebrovasdis.2020.105182

Wang, M., Baker, J. S., Quan, W., Shen, S., Fekete, G., \& Gu, Y. (2020). A Preventive Role of Exercise Across the Coronavirus 2 (SARS-CoV-2) Pandemic. Frontiers in Physiology. https://doi.org/10.3389/ fphys.2020.572718

Woods, J. A., Hutchinson, N. T., Powers, S. K., Roberts, W. O., Gomez-Cabrera, M. C., Radak, Z., et al. (2020). The COVID-19 pandemic and physical activity. Sports Medicine and Health Science, 2(2), 55-64. https://doi.org/10.1016/j.smhs.2020.05.006

Publisher's Note Springer Nature remains neutral with regard to jurisdictional claims in published maps and institutional affiliations. 


\section{Authors and Affiliations}

\section{Zuhal Abasıyanık $^{1,2}$ (D) Merve Kurt ${ }^{1,2}$ (D) Turhan Kahraman²}

Merve Kurt

merveekurtt93@gmail.com

Turhan Kahraman

turhan.kahraman@yahoo.com

1 Graduate School of Health Sciences, Dokuz Eylul University, Izmir, Turkey

2 Department of Physiotherapy and Rehabilitation, Faculty of Health Sciences, Izmir Katip Celebi University, Izmir, Turkey 